\title{
Managing Postural Hypotension in Diabetic Autonomic Dysfunction When Adrenergic Drugs are Contraindicated: Case Report and Review of Literature
}

Syed Rizwan A. Bokhari ${ }^{1}$, Faisal Akhtar ${ }^{2}$, Qurrat-Ul-Ain Abid ${ }^{3}$, Uzma Jahanzaib ${ }^{4}$, Maria R. Bokhari $^{5}$, Sana Hasan ${ }^{6}$, Khurshid Khan ${ }^{7}$

1. Nephrology, Tulane University School of Medicine, New Orleans, USA 2. Neurology, Ochsner Health System, New Orleans, USA 3. Internal Medicine, Jinnah Hospital Lahore (jhl) Pakistan, Broken Bow, PAK 4. Department of Internal Medicine, Division of Endocrinology, Mayo Hospital Lahore 5. Department of Radiology, Tulane University School of Medicine, New Orleans, LA 6. Department of Nephrology and Renal Transplant, Prince Sultan Medical Military City, Riyadh, Riyadh, SAU 7. Jinnah Allama Iqbal Institute of Diabetes and Endocrinology, Allama Iqbal Medical College/Jinnah Hospital, Lahore, PAK

$\square$ Corresponding author: Faisal Akhtar, faisalakhtar121@yahoo.com

Disclosures can be found in Additional Information at the end of the article

\section{Abstract}

Postural hypotension, as a manifestation of autonomic neuropathy is a very sinister long-term debilitating complication of diabetes, is usually irreversible and tough to manage with medications. The treatment of this condition following the standard treatment protocols can be contraindicated in the patients with underlying heart conditions. We report the case of a patient at our hospital who presented with full-blown symptomatic dysautonomia secondary to long-standing diabetes, with bedside testing positive for autonomic dysfunction. Treating this patient with the standard protocol of adrenergic agonist could have worsened his underlying coronary artery disease. So, we moved a step aside to go out of the box and we have a trial of the $\beta 1$-selective beta-blocker, with astonishing results and significant improvement in the quality of life and symptoms of postural hypotension. We report here the use of alternative treatment option in managing a patient with severe postural hypotension secondary to diabetes-related autonomic neuropathy when adrenergic drugs are contraindicated.

\section{Categories: Cardiology, Internal Medicine, Nephrology}

Keywords: diabetic neuropathy, nephrology, cardiology, internal medicine, autonomic neuropathy, dm, blood pressure, beta blockers

Received 12/28/2017

Review began 12/28/2017

Review ended 01/03/2018

Published 01/08/2018

\section{(c) Copyright 2018}

Bokhari et al. This is an open access article distributed under the terms of the Creative Commons Attribution License CC-BY 3.0., which permits unrestricted use, distribution, and reproduction in any medium, provided the original author and source are credited.

\section{Introduction}

Orthostatic hypotension is the core feature of autonomic failure. Orthostatic hypotension is defined as a fall in the blood pressure of $20 / 10 \mathrm{mmHg}$ on standing for three minutes. This definition is meant to capture the initial or mild cases. The symptomatic orthostatic hypotension is more commonly encountered in the subjects with falls in blood pressure that may range from this figure to the severe cases where the fall in the blood pressure may be 100/50 mmHg. The most common symptoms of orthostatic hypotension are lightheadedness, mining of vision, and discomfort in the head (typically occipital), neck, shoulders, and sometimes the chest. Orthostatic hypotension is the most bothering symptom, often complained by the patients suffering from diabetic neuropathy [1]. Many patients with orthostatic hypotension can be treated by increasing the plasma volume and by increasing the 
fluid and sodium intake to improve the orthostatic tolerance or can be treated pharmacologically by giving fludrocortisone with sympathomimetic agents [2].

Diabetic neuropathy can be asymptomatic or can be a cause of significant disability. Any or all the organs innervated by the autonomic system can be affected by diabetes. The symptoms can arise from the involvement of a single system or multiple systems and can range from postural hypotension, diarrhea, gastroparesis, overflow urinary incontinence, impotence to dry skin and gustatory sweating [3]. Commonly instituted tests used to detect the presence of dysautonomia are:

1) Beat-to-beat cardiac variation with position or respiration

2) Valsalva ratio (normal-longest to shortest relative risk $(\mathrm{R}-\mathrm{R})$ ratio $>1.2$ )

3) Heart rate response to deep breathing (normal-E: I ratio $\geqslant 1.17$ )

4) Ratio being 30:15 R-R Interval ratio (30th to 15th R-R ratio on standing > 1.03)

5) Fall in the blood pressure of 20/10 mmHg on standing for three minutes.

\section{Case Presentation}

Diabetic autonomic neuropathy often goes unrecognized and it remains asymptomatic most of the time, but often it presents with debilitating symptoms. We present a case of 48-year-old male with the history of a type-II diabetic for 20 years and coronary artery disease (CAD). The patient had poor control of diabetes and had diabetic complications of gastroparesis, retinopathy, nephropathy, neuropathy, and CAD. The presenting complaints were dizziness for two months with the progressive inability to stand without support. On examination, he was a lethargic middle-aged man lying comfortably in bed. The lying blood pressure (BP) was 150/90 mmHg with the significant postural drop measuring up to $80 / 60 \mathrm{mmHg}$ after two minutes of standing. The neurological exam of diabetes showed distal symmetric sensory neuropathy to light touch, while the vibration sensation and proprioception were intact. The fundoscopy revealed scattered hard exudates, dot, and blot hemorrhages. He was never tested for autonomic dysfunction prior to this encounter and did not take any medication, since midodrine was not available in Pakistan and fludrocortisone was never prescribed by the general practitioner, due to the lack of complete testing and affordability issues.

\section{Electrocardiography findings suggestive of dysautonomia were:}

1) Abnormal heart rate response to deep breathing was 1.0 (normal-E: I ratio $\geqslant 1.17$ )

2) The abnormal Valsalva ratio was 1.03 (normal-longest to shortest R-R ratio > 1.2)

3) Abnormal 30:15 R-R interval ratio was 0.99 (30th to 15th, R-R interval ratio on standing > 1.03).

These three tests along with the significant postural drop were suggestive of marked cardiac dysautonomia in this patient. The lab data showed the complete blood count, serum electrolyte test, renal function tests, liver function tests, serum cortisol, Adrenocorticotropic hormone (ACTH) and the thyroid function tests as normal. Midodrine, in this patient, could not be used because of the history of the significant CAD. The patient did not have any response to fludrocortisone. So as a final resort, he was started on atenolol P.O. (initial dose of atenolol 
$12.5 \mathrm{mg}$ b.i.d. which was gradually increased to $25 \mathrm{mg}$ b.i.d.). The patient had dramatic clinical improvement with this therapy. The orthostatic blood pressure changes improved and he was able to ambulate without any difficulty.

\section{Discussion}

Diabetic autonomic neuropathy is the impairment of autonomic control in the setting of diabetes after exclusion of other causes. Ewing, et al. recommend five simple tests, the cardiac autonomic reflex tests to establish the diagnosis: 1) Heart rate variability (HRV) with deep breathing; 2) the HRV lying to standing; 3) the Valsalva maneuver; 4) postural fall in the blood pressure; and 5) the blood pressure response to sustained handgrip [4]. A single abnormal test may indicate early cardiac autonomic neuropathy and three positive tests are recommended for a definite diagnosis. The diabetic autonomic neuropathy can be quite challenging and difficult to treat. Although medications cannot cure autonomic neuropathy, they can help with finding the symptoms.

\section{Drugs used for the treatment of postural hypotension}

Fludrocortisone

Fludrocortisone is a mineralocorticoid, which causes sodium retention and enhances the sensitivity of the blood vessels to circulating catecholamine [5].

\section{Midodrine}

Midodrine is a prodrug that acts by stimulating the alpha-1 adrenoreceptors to constrict the blood vessels and raise the blood pressure [6]. The pressor effect of midodrine comes on within about 30 minutes and wanes after four or five hours.

Even though adrenergic drugs like midodrine are the first line choice in the patients with orthostatic hypotension from diabetic autonomic neuropathy, their use is limited in the patients with contraindications.

The standard protocol of treating diabetic autonomic neuropathy related postural hypotension recommends using fludrocortisones and midodrine, however, our patient was having coronary artery disease, so the use of adrenergic agonist was just synonymous to relieving one debilitating symptom and putting him into another life-threatening state. The studies have shown that the beta-adrenergic blockers do not cause orthostatic hypotension [7]. A cardioselective $\beta 1$-blocker, atenolol $12.5 \mathrm{mg}$ was started twice daily, which was gradually increased to $25 \mathrm{mg}$ b.i.d. After some time, the patient reported significant improvement in his postural hypotension.

Nonselective $\beta$-blockers with intrinsic sympathomimetic activity and the $\beta 1$ selective agents with no intrinsic sympathomimetic activity like atenolol have been used in several trials in the treatment of orthostatic hypotension and for the prevention of presyncope with variable results [8]. The suggested mechanism of action of these agents is the blockade of the $\beta 2$ receptors, allowing unopposed adrenoreceptor mediated vasoconstriction [7]. Moreover, the beta-blockers can help regulate the heart rate and rhythm with beneficial effects for the patients with coronary artery disease and ischemic heart disease [9]. However, we noticed a remarkable role of the beta-blockers in the treatment of postural hypotension due to diabetic autonomic dysfunction.

A similar case was reported in the Journal of Human Hypertension in 2006. A 54-year-old male 
with diabetes was referred to the hypertension clinic in the Jichi Medical School Hospital with symptoms of dizziness and orthostatic intolerance and was successfully treated with the $\beta 1$ blocker [10].

The $\beta 1$ - selective blocker (atenolol) effectively reduced the orthostatic blood pressure changes in this patient with severe orthostatic hypotension due to diabetic autonomic neuropathy and the main finding was an improvement of the elevated supine blood pressure without reducing the standing blood pressure. According to the author, this differential antihypertensive effect is ideal for the medication in orthostatic hypotension, but the authors found it to be very different from other case reports, of the effects of the $\beta$-blockers, where the emphasis has been on increasing the standing pressure. They claim that the study was unique in two ways: 1 ) the use of 24-hr ambulatory monitoring and 2) they describe a potentially different mechanism whereby the $\beta$-blockers may benefit the patients with orthostatic hypotension.

\section{Conclusions}

Postural hypotension, as part of the spectrum of diabetic autonomic dysfunction, can be difficult to manage in conditions where adrenergic drugs are contraindicated. Our case report highlighted the importance of the beta blockers for the treatment of postural hypotension in special circumstances. Increased awareness and effective monitoring of diabetic autonomic neuropathy can help decrease the morbidity associated with it. Further studies are needed to address this cumbersome condition.

\section{Additional Information \\ Disclosures}

Human subjects: Consent was obtained by all participants in this study. Conflicts of interest: In compliance with the ICMJE uniform disclosure form, all authors declare the following: Payment/services info: All authors have declared that no financial support was received from any organization for the submitted work. Financial relationships: All authors have declared that they have no financial relationships at present or within the previous three years with any organizations that might have an interest in the submitted work. Other relationships: All authors have declared that there are no other relationships or activities that could appear to have influenced the submitted work.

\section{References}

1. Metzler M, Duerr S, Granata R, et al.: Neurogenic orthostatic hypotension: Pathophysiology, evaluation, and management. J Neurol. 2013, 260:2212-2219. 10.1007/s00415-012-6736-7

2. Freeman R: Treatment of orthostatic hypotension. Semin Neurol. 2003, 23:435-442. $10.1055 /$ s-2004-817727

3. Duby JJ, Campbell RK, Setter SM, et al.: Diabetic neuropathy: An intensive review. Am J Health Syst Pharm. 2004, 61:160-173.

4. Ewing DJ, Borsey DQ, Bellavere F, et al.: Cardiac autonomic neuropathy in diabetes: Comparison of measures of R-R interval variation. Diabetologia. 1981, 21:18-24. 10.1007/BF03216217

5. Oldenburg O, Kribben A, Baumgart D, et al.: Treatment of orthostatic hypotension . Curr Opin Pharmacol. 2002, 2:740-747. 10.1016/S1471-4892(02)00213-8

6. Kaufmann H, Brannan T, Krakoff L, et al.: Treatment of orthostatic hypotension due to autonomic failure with a peripheral alpha-adrenergic agonist (midodrine). Neurology. 1988, 38:951. 10.1212/WNL.38.6.951

7. Frohlich ED, Tarazi RC, Dustan HP, et al.: The paradox of beta-adrenergic blockade in hypertension. Circulation. 1968, 37:417-423. 10.1161/01.CIR.37.3.417

8. Lamarre-Cliche M: Drug treatment of orthostatic hypotension because of autonomic failure or neurocardiogenic syncope. Am J Cardiovasc Drugs. 2002, 2:23-35. 10.2165/00129784- 


\section{Cureus}

200202010-00004

9. Niemela MJ, Airaksinen KEJ, Huikuri HV: Effect of Beta-blockade on heart rate variability in patients with coronary artery disease. J Am Coll Cardiol. 1994, 23:1370-1377. 10.1016/07351097(94)90379-4

10. Eguchi K, Pickering TG, Ishikawa J, et al.: Severe orthostatic hypotension with diabetic autonomic neuropathy successfully treated with a $\beta 1$-blocker: A case report. J Hum Hypertens. 2006, 20:801-803. 10.1038/sj.jhh.1002066 